\title{
162-LB: Plasma Glycated CD59 Predicts Postpartum Glucose Intolerance after Gestational Diabetes
}

KATRIEN BENHALIMA, DONGDONG MA, MICHELLE TOTH CASTILLO MATHIEU, JOSE HALPERIN

Diabetes 2021;70(Supplement_1):162-LB https://doi.org/10.2337/db21-162-LB

pGCD59 is an emerging biomarker for diabetes and GDM. In studies of women undergoing GDM screening either early in pregnancy (week <20) or at pregnancy weeks 24-28, pGCD59 classified GDM subjects with high sensitivity and specificity and was associated with the prevalence of large for gestational age (LGA) babies (Diabetes Care 2017 and JCEM 2020). GDM developed early in pregnancy imparts a seven-fold increased risk of subsequent glucose intolerance and diabetes. Since $\mathrm{HbA} 1 \mathrm{c}$ is not sensitivity to detect glucose intolerance in early postpartum, professional organizations including ADA, recommend that women with GDM should undergo OGTT testing 6-12 weeks postpartum. However, the cumbersome nature of OGTT makes compliance with this requirement very low, highlighting the need for alternative biomarkers that can be used postpartum to test women with GDM for potential conversion glucose intolerance. To assess the ability of pGCD59 to identify postpartum conversion to glucose intolerance in women with GDM, levels of pGCD59 were measured in samples from participants in the Belgian Diabetes in Pregnancy (BEDIP-N, Diabetes Care 2018) cohort study who underwent OGTT testing 6-12 weeks postpartum. Among 105 samples from women with GDM, 36 subjects met OGTT criteria for glucose intolerance (cases) and 70 had normal OGTT (matched controls). Maternal age, race/ethnicity, and BMI were similar among Cases and Controls. pGCD59 was significantly higher in cases than in controls ( $1.5 \pm 0.6 \mathrm{SPU}$ vs. $1.0 \pm 0.6 \mathrm{SPU}, \mathrm{p}<0.001)$ and accurately predicted the postpartum conversion to glucose intolerance as indicated by an AUCROC of 0.72 (95\% Cl 0.62-0.83). A 0.5unit increase in postpartum pGCD59 was associated with an OR of 2.0 (95\% Cl 1.342.95, $p<0.001$ ) for glucose intolerance. Our results indicate that pGCD59 is a simpler biomarker to assess postpartum conversion to glucose intolerance in women with GDM and represents an alternative to improve compliance with postpartum screening for glucose intolerance.

\section{Disclosure}

K. Benhalima: None. D. Ma: None. M. Toth castillo: None. A. Laenen: None. C. Mathieu: Advisory Panel; Self; Novo Nordisk, Sanofi, Merck Sharp and Dohme Ltd., Eli Lilly and Company, Novartis, AstraZeneca, Boehringer Ingelheim, Roche, Medtronic, ActoBio Therapeutics, Pfizer, Insulet and Zealand Pharma, Research Support; Self; Medtronic, Novo Nordisk, Sanofi and ActoBio Therapeutics, Speaker's Bureau; Self; Novo Nordisk, Sanofi, Eli Lilly and Company, Boehringer Ingelheim, AstraZeneca and Novartis. J. Halperin: Board Member; Self; Mellitus, LLC.

Funding 
National Institutes of Health (R01DK118528, DK101442); Belgian National Lottery; Fund of Academic Studies of UZ Leuven; Yvonne and Jacques François-de Meurs of the King Boudewijn Foundation 